\title{
Duration of noninvasive ventilation is not a predictor of clinical outcomes in patients with acute exacerbation of COPD and respiratory failure
}

\author{
Laith Ghazala ${ }^{1,2}$, Umur Hatipoğlu' ${ }^{1}$, Tanya Devnani ${ }^{1}$, Erin Covert ${ }^{3}$, Justin Hanks ${ }^{3}$, Katelyn Edwards ${ }^{3}$, Maeve Macmurdo ${ }^{1}$, Manshi Li $^{4}$, \\ Xiaofeng Wang ${ }^{4}$, Abhijit Duggal ${ }^{1}$
}

\begin{abstract}
L Ghazala, U Hatipoğlu, T Devnani, E Covert, J Hanks. Duration of noninvasive ventilation is not a predictor of clinical outcomes in patients with acute exacerbation of COPD and respiratory failure. Can J Respir Ther 2021;57:113-118. doi: 10.29390/cjrt-2021-021.

Purpose: Acute exacerbation of chronic obstructive pulmonary disease (COPD) is a major cause of mortality and morbidity. Noninvasive ventilation (NIV) is proven to be effective in the majority of patients with acute exacerbation COPD (AECOPD) complicated with respiratory failure. NIV could be lifesaving but also can delay mechanical ventilation if its efficacy is not assessed in a timely manner. In this study, we analyzed potential predictors of NIV failure in AECOPD in a tertiary medical intensive care unit (MICU). In particular, we wondered whether duration of NIV among those who eventually failed was associated with poor outcomes.

Methods: A retrospective review of consecutive patients with a primary diagnosis of AECOPD requiring NIV admitted to the MICU was conducted for the period between 2012 and 2017. Baseline data included demographics, APACHE III score, albumin level, blood lactate, and blood gas elements. Additional chart review was performed to collect NIV setting parameters on presentation to the MICU. Clinical outcome variables collected included outcome and duration of NIV, duration of invasive mechanical ventilation, MICU length of stay, hospital length of stay, and in-hospital mortality. Multivariate regression analysis was performed to determine independent variables associated with clinical outcomes.

Results: There were 370 patients who met the inclusion criteria; $53.2 \%$ were male. Mean age was $64.7 \pm 11.2$ years old. Mean baseline $\mathrm{FEV}_{1}$ was $34 \pm 17 \%$ of predicted. Patients had mean pH of $7.20 \pm 0.54$ and $\mathrm{P}_{\mathrm{a}} \mathrm{CO}_{2}$ of $70.3 \pm 28.7$ on presentation; 323 patients (87.3\%) were successfully weaned off NIV; 47 patients (12.7\%) failed NIV and required invasive mechanical ventilation. APACHE III score was higher among patients who failed NIV (68.3 \pm 18.9 versus $48.8 \pm 15.2, P<0.001)$. In the subset of 47 patients who failed NIV requiring intubation, duration of NIV was $25.0 \pm 58.8$ h. Multivariate regression analysis yielded a model consisting of APACHE III score and body mass index as predictive variables for NIV failure (C-statistic $=0.809)$. Duration of NIV was not associated with worse clinical outcomes among patients who failed NIV.

Conclusions: NIV is successful in preventing invasive mechanical ventilation in majority of patients with acute respiratory failure due to COPD. Patients with worse clinical status at presentation are more likely to fail NIV and require mechanical ventilation. In the subgroup of patients who failed NIV, duration of NIV prior to intubation was not associated with poor clinical outcomes.
\end{abstract}

Key Words: COPD; hypercapneic respiratory failure; noninvasive ventilation; outcome predictors

\section{INTRODUCTION}

Noninvasive ventilation (NIV) is the preferred initial modality in providing ventilator assistance to patients with acute hypercapnic respiratory due to acute exacerbation of chronic obstructive pulmonary disease (AECOPD) [1]. The use of NIV in patients with AECOPD has been shown to improve survival and decrease need for subsequent mechanical ventilation [2, 3]. While use of NIV for this indication is increasingly common, some patients with AECOPD will ultimately deteriorate despite use of NIV, eventually requiring invasive mechanical ventilation (IMV) [4]. Furthermore, a lack of response to NIV use may also be associated with additional harms mainly in the peri-intubation period [4]. Therefore, to minimize potential harms, predicting which patients with AECOPD are at high risk of NIV failure related to potential harm of prolonged NIV has been an intense area of research.
Based on current literature between $9 \%$ and $50 \%$ of patients presenting with AECOPD fail to respond to NIV. Previous studies have focused on identifying factors associated with success of NIV in AECOPD patients. Studies have identified presence of concomitant hypoxemia and improvement in heart rate, respiratory rate, and hypercapnia after $1 \mathrm{~h}$ of NIV treatment, as predictors of successful NIV use in AECOPD $[5,6]$. Duration of NIV is commonly cited as a risk for peri-intubation related harm [4]. Notwithstanding, a prospective study showed no difference in outcomes between COPD patients who were maintained on NIV despite persistent hypercapnia and those who responded early [7]. Whether duration of NIV itself is a predictor of prolonged IMV or mortality remains unclear.

In this study, we evaluated patients with a diagnosis of AECOPD who were admitted to a tertiary medical intensive care unit (MICU) for

\footnotetext{
${ }^{1}$ Respiratory Institute, Cleveland Clinic, Cleveland, OH, USA

${ }^{2}$ Trillium Health Partners, Mississauga, ON, Canada

${ }^{3}$ Internal Medicine, Cleveland Clinic, Cleveland, OH, USA

${ }^{4}$ Quantitative Health Sciences, Cleveland Clinic, Cleveland, OH, USA

Correspondence: Laith Ghazala, Pulmonary and Critical Care Medicine, Cleveland Clinic, 9500 Euclid Ave, A9, Cleveland, OH 44106, USA. Tel: 905 699 3086,E-mail: laithgazala@gmail.com
}

Published online at https://www.cjrt.ca on 18 August 2021

This open-access article is distributed under the terms of the Creative Commons Attribution Non-Commercial License (CC BY-NC) (http:// creativecommons.org/licenses/by-nc/4.0/), which permits reuse, distribution and reproduction of the article, provided that the original work is properly cited and the reuse is restricted to noncommercial purposes. For commercial reuse, contact editor@csrt.com 
the treatment of acute hypercapnic respiratory failure with NIV. We aimed to delineate early predictors of NIV failure and specifically assess whether prolonged NIV duration is a predictor of poor clinical outcomes, including prolonged mechanical ventilation and death.

\section{METHODS}

We conducted a retrospective cohort study in a tertiary MICU. The study was approved under the institute Institutional Review Board number (18-457). Utilizing a pre-existing APACHE database, patients admitted between January of 2012 and December of 2017 with a diagnosis of COPD were identified. All the patients were then screened using the APACHE database coding for a primary admission diagnosis of AECOPD, needing NIV and having an age greater than 18 years. Those who met the inclusion criteria underwent chart revision. A documented use of NIV with the initial settings recorded in the respiratory therapist data set was required for inclusion in the study. Patients who were intubated on arrival to the MICU, received NIV for a reason other than AECOPD, or had pre-existing advanced directives against mechanical ventilation were excluded from further analysis (Figure 1). 10\% of the charts were randomly selected and were audited by the first author to confirm the accuracy of data obtained from respiratory therapy and APACHE databases.

We collected demographic data and patient characteristics including age, sex, smoking status, serum albumin, body mass index (BMI), home oxygen utilization, and the presence or absence of co-existing obstructive

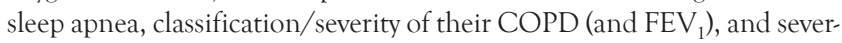
ity of illness on presentation to the MICU (APACHE III Score). Arterial blood gases taken at the time of ICU admission as well as after implementation of NIV were recorded to examine serial changes in $\mathrm{pH}$, $\mathrm{P}_{\mathrm{a}} \mathrm{CO}_{2}, \mathrm{P}_{\mathrm{a}} \mathrm{O}_{2}$, and lactate. NIV driving pressure (defined as inspiratory positive airway pressure minus expiratory positive airway pressure) on initiation of NIV was also recorded.

The primary outcome for this study was rate of failure of NIV with need for IMV. Secondary outcomes included duration of NIV, duration of mechanical ventilation, successful extubation (defined as extubation without the need for re-intubation within the index MICU admission), MICU length of stay, hospital length of stay, and in-hospital mortality.

\section{Statistical analysis}

ANOVA was used to compare continuous variables between the two groups, and Pearson's chi-square test was used to compare categorical variables. Logistic and linear regression models were performed for binary and continuous outcomes separately. Variables were considered significant at $P<0.15$ on univariate analysis and subsequently identified as potential predictor variables and entered into a multivariate regression model. Correlation analysis of the predictors was conducted to avoid the multi-collinearity in a regression model. Stepwise variable selection procedure was then performed to identify the final multivariate regression model with a significant subset of predictors. Receiver operator characteristics curves were constructed for logistic regression models. SAS 9.4 software (SAS Institute, Cary, NC) was used for all analyses.

\section{RESULTS}

Between 1 January 2012 and 31 December 2017, 25,913 patients received care in the MICU; 754 patients were admitted to the MICU with a primary diagnosis of COPD. In this subgroup, 370 patients met the inclusion criteria and were included in the analysis (Figure 1); $323(87.3 \%)$ patients did not require subsequent IMV (NIV success group); 47 patients failed initial support with NIV and underwent endotracheal intubation (NIV failure group).

\section{FIGURE 1.}

Flowchart for patient selection. ICU = intensive care unit; NIV = noninvasive ventilation; COPD = chronic obstructive pulmonary disease.

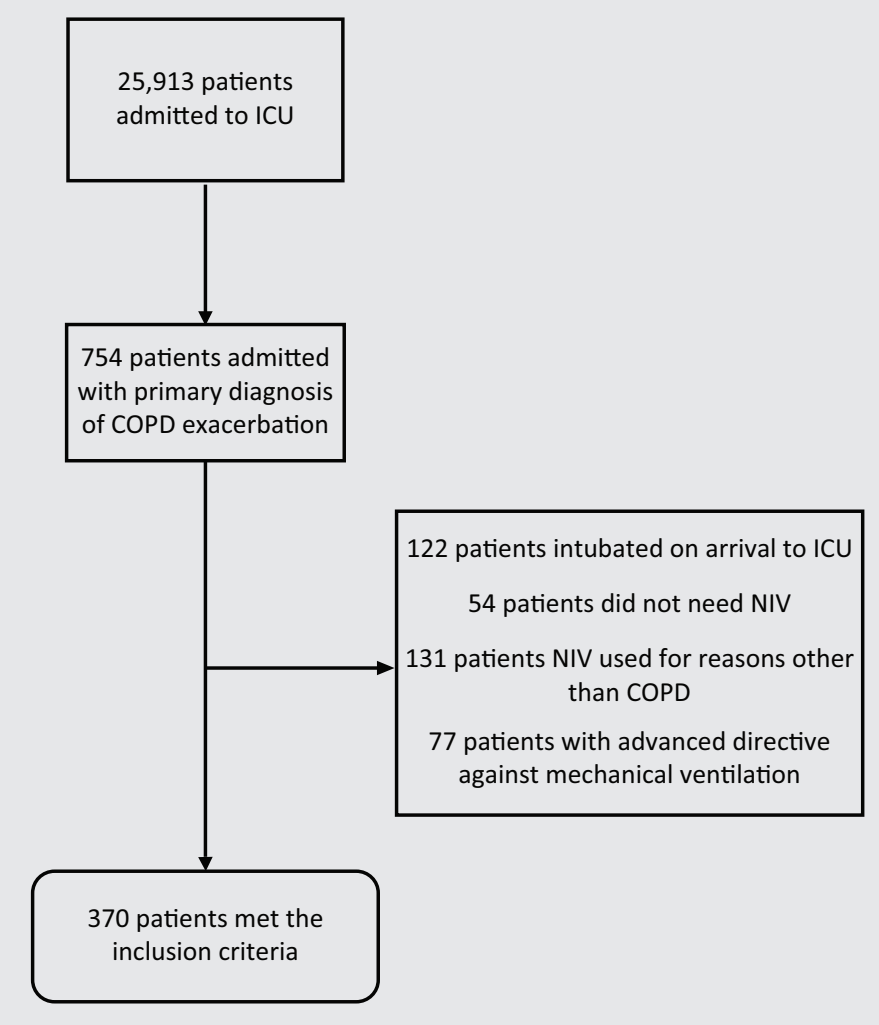


There were no significant differences in patient age, sex, or smoking status between the two groups (Table 1). Compared with patients who were able to successfully wean from NIV without the need for mechanical ventilation, patients who required IMV had higher APACHE III score on admission ( 68.3 vs. $48.8, P<0.001)$. We found no statistically significant differences in $\mathrm{FEV}_{1}$ percent predicted at baseline, serum albumin levels, baseline NIV driving pressure, and other tested variables between the two groups (Table 1).
Clinical outcomes including duration of invasive and NIV are displayed in Table 2. Median duration of NIV use trended lower in the NIV success group without statistical significance $(16.2 \pm 33.2 \mathrm{~h}$ versus $25.0 \pm 58.8 \mathrm{~h}$ in the success and failure groups, respectively, $P=0.14$ ). Hospital and MICU length of stay (LOS) were significantly longer in the NIV failure group (Table 2).

In the univariate analysis APACHE III score was significantly associated with NIV success (OR 0.936, 95\% CI 0.917-0.955, $P<0.0001$ )

\section{TABLE 1}

Patient characteristics

\begin{tabular}{|c|c|c|c|c|c|c|c|}
\hline \multirow[b]{2}{*}{ Factor } & \multicolumn{2}{|c|}{ Overall $(n=370)$} & \multicolumn{2}{|c|}{ Success $(n=323)$} & \multicolumn{2}{|c|}{ Fail $(n=47)$} & \multirow[b]{2}{*}{$P$} \\
\hline & $n$ & Statistics & $n$ & Statistics & $n$ & Statistics & \\
\hline Age & 370 & $64.7 \pm 11.2$ & 323 & $64.7 \pm 11.3$ & 47 & $64.5 \pm 10.9$ & $0.91^{\mathrm{a}}$ \\
\hline Sex & 370 & - & 323 & - & 47 & - & $0.12^{b}$ \\
\hline Female & - & $173(46.8)$ & - & $146(45.2)$ & - & $27(57.4)$ & - \\
\hline Male & - & $197(53.2)$ & - & $177(54.8)$ & - & $20(42.6)$ & - \\
\hline Body mass index & 367 & $28.2 \pm 10.2$ & 322 & $27.8 \pm 9.7$ & 45 & $30.7 \pm 13.0$ & $0.075^{a}$ \\
\hline Current smoker & 337 & - & 298 & - & 39 & - & $0.29^{b}$ \\
\hline No & - & $232(68.8)$ & - & $208(69.8)$ & - & $24(61.5)$ & - \\
\hline Yes & - & $105(31.2)$ & - & $90(30.2)$ & - & $15(38.5)$ & - \\
\hline Home Oxygen & 332 & - & 292 & - & 40 & - & 0.97 \\
\hline No & - & $74(22.3)$ & - & $65(22.3)$ & - & $9(22.5)$ & - \\
\hline Yes & - & $258(77.7)$ & & $227(77.7)$ & - & $31(77.5)$ & - \\
\hline APACHE III score & 369 & $51.3 \pm 16.9$ & 322 & $48.8 \pm 15.2$ & 47 & $68.3 \pm 18.9$ & $<0.001^{\mathrm{a}}$ \\
\hline Baseline_FEV 1 & 265 & $0.34 \pm 0.17$ & 234 & $0.33 \pm 0.16$ & 31 & $0.38 \pm 0.21$ & $0.18^{a}$ \\
\hline Baseline respiratory rate & 368 & $23.5 \pm 6.1$ & 321 & $23.3 \pm 6.0$ & 47 & $24.9 \pm 7.2$ & $0.11^{\mathrm{a}}$ \\
\hline Albumin & 307 & $3.6 \pm 0.59$ & 266 & $3.6 \pm 0.58$ & 41 & $3.5 \pm 0.66$ & $0.12^{\mathrm{a}}$ \\
\hline No & - & $62(16.8)$ & - & $48(14.9)$ & - & $14(29.8)$ & - \\
\hline Yes & - & $308(83.2)$ & - & $275(85.1)$ & - & $33(70.2)$ & - \\
\hline $\mathrm{pH}$ & 308 & $7.2 \pm 0.54$ & 275 & $7.2 \pm 0.57$ & 33 & $7.3 \pm 0.09$ & $0.96^{a}$ \\
\hline $\mathrm{P}_{\mathrm{a}} \mathrm{CO}_{2}$ & 308 & $70.3 \pm 28.7$ & 275 & $69.5 \pm 28.9$ & 33 & $76.8 \pm 26.8$ & $0.17^{\mathrm{a}}$ \\
\hline Baseline lactate & 177 & $1.4 \pm 1.2$ & 145 & $1.4 \pm 1.01$ & 32 & $1.7 \pm 1.8$ & $0.26^{a}$ \\
\hline Driving pressure & 363 & $9.1 \pm 3.4$ & 317 & $9.0 \pm 3.3$ & 46 & $9.3 \pm 3.7$ & $0.56^{a}$ \\
\hline
\end{tabular}

${ }^{a}$ Two-sample t-test.

${ }^{b}$ Pearson's chi-square test.

\section{TABLE 2}

Clinical outcomes and duration of mechanical ventilation

\begin{tabular}{|c|c|c|c|c|c|c|c|}
\hline & \multicolumn{2}{|c|}{ Overall $(n=370)$} & \multicolumn{2}{|c|}{ Success $(n=323)$} & \multicolumn{2}{|c|}{ Fail $(n=47)$} & \multirow[b]{2}{*}{$P$} \\
\hline & $n$ & Statistics & $n$ & Statistics & $n$ & Statistics & \\
\hline Medical intensive care unit LOS & 369 & $3.4 \pm 4.7$ & 322 & $2.6 \pm 2.9$ & 47 & $9.0 \pm 8.9$ & $<0.001^{\mathrm{a}}$ \\
\hline Hospital LOS & 370 & $10.6 \pm 14.6$ & 323 & $9.6 \pm 14.8$ & 47 & $17.6 \pm 11.5$ & $<0.001^{\mathrm{a}}$ \\
\hline Duration IMV & 47 & $115.1 \pm 130.8$ & 0 & - & 47 & $115.1 \pm 130.8$ & - \\
\hline Duration NIV & 361 & $17.3 \pm 37.4$ & 316 & $16.2 \pm 33.2$ & 45 & $25.0 \pm 58.8$ & $0.14^{\mathrm{a}}$ \\
\hline In-hospital mortality & 370 & - & 323 & - & 47 & - & $0.029^{b}$ \\
\hline No & - & $358(96.8)$ & - & $315(97.5)$ & - & $43(91.5)$ & - \\
\hline Yes & - & $12(3.2)$ & - & $8(2.5)$ & - & $4(8.5)$ & - \\
\hline Able to extubate & 47 & - & 0 & - & 47 & - & - \\
\hline No & - & $5(10.6)$ & - & - & - & $5(10.6)$ & - \\
\hline Yes & - & $42(89.4)$ & - & - & 一 & $42(89.4)$ & - \\
\hline
\end{tabular}

${ }^{a}$ Two-sample t-test.

${ }^{b}$ Pearson's chi-square test.

$N I V=$ Noninvasive ventilation $;$ LOS = length of stay; IMV = invasive mechanical ventilation 
(Table 3). Multivariable logistic regression model showed that combining APACHE III score and BMI (Table 4) had the highest accuracy for predicting likelihood of NIV failure (c-statistic 0.809). (Figure 2).

\section{DISCUSSION}

In this single academic center cohort, $87 \%$ of the patients with AECOPD admitted to the MICU improved with the application of NIV alone and were able to avoid IMV. We found no significant differences in demographic variables or baseline lung impairment between the patients who failed NIV and those who did not. Failure of NIV was predominantly

\section{TABLE 3}

Univariable logistic regression model for noninvasive ventilation success

\begin{tabular}{|c|c|c|c|}
\hline Variable & Odds ratio & $\begin{array}{c}95 \% \text { confidence } \\
\text { interval }\end{array}$ & $P$ \\
\hline Baseline respiratory rate & 0.963 & $0.918-1.009$ & 0.1161 \\
\hline APACHE III score & 0.936 & $0.917-0.955$ & $<0.0001$ \\
\hline Baseline lactate & 0.854 & $0.646-1.128$ & 0.2663 \\
\hline Albumin & 1.503 & $0.897-2.518$ & 0.1219 \\
\hline Home Oxygen (yes vs no) & 1.014 & $0.459-2.238$ & 0.9727 \\
\hline Driving pressure & 0.974 & $0.890-1.065$ & 0.5609 \\
\hline Body mass index & 0.976 & $0.950-1.003$ & 0.0790 \\
\hline $\mathrm{PaCO}_{2}$ & 0.993 & $0.983-1.003$ & 0.1772 \\
\hline $\mathrm{pH}$ & 0.981 & $0.488-1.971$ & 0.9561 \\
\hline Baseline FEV1 & 0.251 & $0.032-1.939$ & 0.1851 \\
\hline Lung compliance & 1.000 & $0.992-1.008$ & 0.9628 \\
\hline
\end{tabular}

driven by a higher APACHE III score at admission and a model combining APACHE III score and BMI discriminated successfully between success and failure of NIV. Notably, we found no effect of the duration of NIV on adverse outcomes. To our knowledge, this is the largest cohort study from an academic setting for the investigation of the effect of NIV duration on clinical outcomes.

Failure of NIV has been associated with poor clinical outcomes overall. Walkey and Soylemez-Wiener [8] reported a greater odds of hospital mortality for 54,911 patients hospitalized with acute respiratory failure due to COPD who failed NIV prior to IMV in the Nationwide Inpatient Sample between 2000 and 2009 (multivariate adjusted OR 1.14; 95\% CI 1.11-1.17). Similarly, Correa and colleagues [9] found that odds of in-hospital mortality was increased by more than 4 times among 85 patients who failed NIV for acute respiratory failure due to COPD. The predictors of NIV failure included age and APACHEII score in this cohort. More recently, a prospective study by Steriade et al. [10] demonstrated a positive association of NIV failure with in-hospital mortality, LOS and duration of NIV in 89 patients with AECOPD, with an overall mortality rate of $11.2 \%$ and a NIV failure rate of $12.4 \%$. The study showed that the total duration of NIV applied within the first $72 \mathrm{~h}$ (mean $30.3 \pm 14.5 \mathrm{~h}$ ) had no impact on NIV failure or mortality. Duan and colleagues [11] derived and

\section{TABLE 4}

Multivariable logistic regression model for noninvasive ventilation success

\begin{tabular}{lccc} 
Variable & Odds ratio & 95\% confidence interval & $\boldsymbol{P}$ \\
\hline APACHE III score & 0.929 & $0.909-0.950$ & $<0.0001$ \\
Body mass index & 0.962 & $0.928-0.996$ & 0.0288 \\
\hline
\end{tabular}

\section{FIGURE 2.}

Receiver operating characteristic curve for the prediction of noninvasive ventilation failure using a model incorporating patient body mass index and APACHE III score. ROC = receiver operator characteristics.

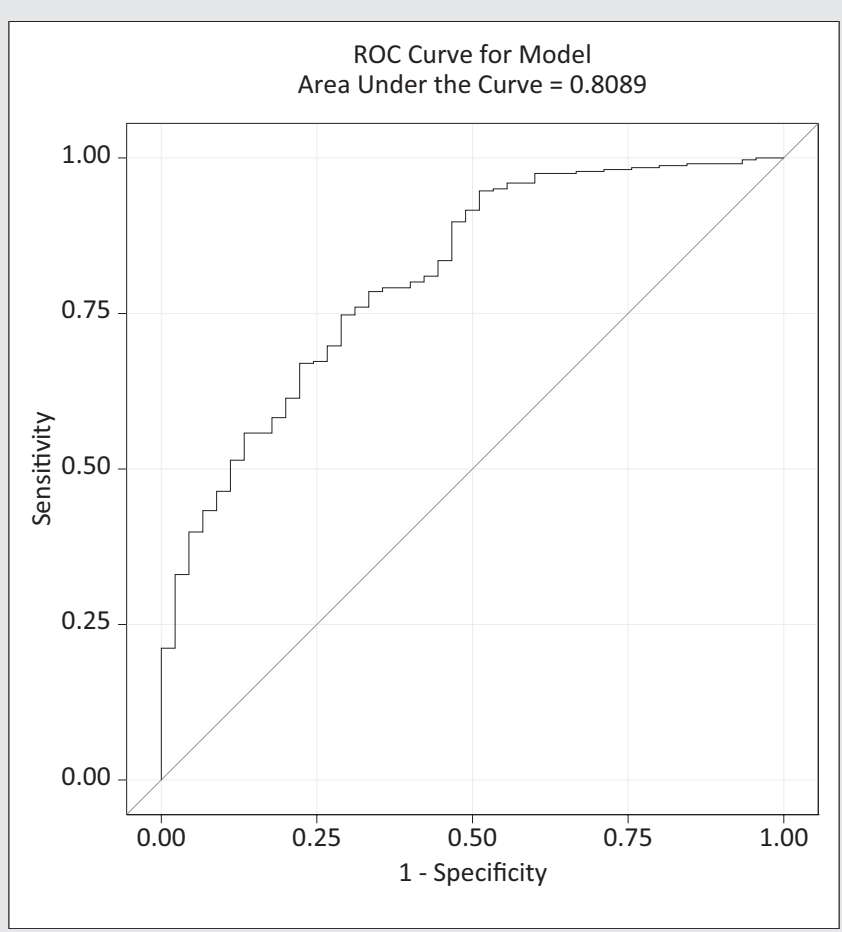


validated a simple risk score that incorporated heart rate, $\mathrm{pH}$, consciousness, oxygenation, and respiratory rate (HACOR) with excellent predictive power for NIV failure among COPD patients .

Our findings confirm and extend these observations. While the ideal duration of NIV prior to initiation of IMV is not clearly defined, our data support the contention that for selected patients, a longer trial of NIV may be appropriate, and may not be associated with the risk of a prolonged ventilator course and poor outcomes if failure ultimately occurs. We should note that these conclusions hold within the context of a MICU with 24-h staffing and an integrated intensivist and respiratory therapist team that manage patients on NIV. Consequently, clinical signs of NIV failure such as tachycardia [12], tachypnea [11, 12], persistent or worsening acidosis [11, 12], decreased consciousness [11, 12], and poor oxygenation [11] are constantly monitored and patients can be easily transitioned to IMV if needed. To that end, delivery of NIV by a dedicated NIV team has been associated with a lower risk of requiring IMV and in-hospital mortality [13].

Our findings are also consistent with Duan and colleagues [11] since APACHE III score captures all elements of HACOR. In our study cohort, elevated BMI was also an independent predictor of NIV failure. BMI has been shown to have a significant nonlinear relationship with overall mortality in COPD. Studies have demonstrated increasing mortality for BMI < 20 and decreasing up to BMI of 30, with association less clear above this threshold [14]. Paradoxically, while mortality has been shown to decrease above BMI of 20, increasing obesity class in COPD has been independently associated with worse quality of life, reduced 6-min walk distance, increased dyspnea, and greater odds of severe AECOPD, possibly from overlapping comorbidities common in this population [14]. The literature regarding the association of NIV failure and BMI is mixed. Steraide et al found that BMI influenced NIV settings, but did not alter NIV failure rates or NIV associated mortality [10]. Conversely, Demoule et al. [15] found that a moderately increased BMI in patients presenting with AECOPD was associated with NIV success. The correlation of BMI with nutrition and muscle mass and the distribution of body fat and its relation to likelihood of NIV failure would benefit from further investigation.

We acknowledge that our study has limitations inherent to its retrospective nature and single-centre origin. It is difficult to determine whether other undocumented or unrecognized factors drive NIV success or failure due to the reliance on data collection on presentation. Initial ventilator settings, and arterial blood gas (ABG) data were occasionally missing on retrospective chart review, which could have provided further insight into reasons for NIV failure. Intubation is a clinical decision and threshold to intubate may vary from provider to provider [16]. Since our MICU is staffed by board-certified intensivists on weekly rotations and the study represents a 6-year time period, we doubt the presence of a significant provider-based bias in the assessment of the need for intubation. Prolonged use of NIV can be associated with adverse events related to the delivery of NIV such as aspiration, abdominal bloating, and skin ulcers. However, NIV is typically well tolerated in patients with AECOPD and requires close supervision [17].

Acute COPD exacerbations can have heterogeneous etiology [18] with potential implications for therapy [19]. Our study did not assess the different exacerbation phenotypes on the outcomes of NIV and therefore cannot exclude a potential effect. Further, our data set did not contain comorbidities beyond those included in the APACHE III scoring system. Inclusion of cardiac comorbities, in particular, may have improved accuracy of the prediction model. Additionally, the relatively low mortality and NIV failure rates seen within our cohort may limit our ability to produce a more precise estimate of the likelihood of NIV failure. The external validity of these findings are limited to the setting of an academic MICU with intensive staffing.

\section{CONCLUSION}

NIV has an established role in the management of acute respiratory failure due to COPD exacerbation even in critically ill patients. In this context, duration of NIV was not associated with worsening outcomes in those who failed NIV and required endotracheal intubation in this large retrospective cohort. Rather, severity of critical illness and BMI predicted NIV failure. These findings support the prolonged use of NIV for acute respiratory failure during AECOPD provided the availability of close monitoring by an ICU team throughout the clinical course.

\section{Contributors}

\section{DISCLOSURES}

L.G: Primary author. Guarantor for this study including data and analysis. Had substantial contributions to the design of the work, the acquisition, analysis, and interpretation of data for the work. Drafting the work and revising it. Had the final approval of the version to be published. E.C, J.H, K.E, T.D: contributed substantially to the study design, data collection and the writing of the manuscript. All approved the final version of the study. M.M, A.D, U.H: contributed substantially to the study design, data analysis and interpretation, and the writing of the manuscript. All approved the final version of the study. M.L, X.W: contributed substantially to the study design and data analysis. All approved the final version of the study.

\section{Funding}

This study did not receive any specific grant from funding agencies in the public, commercial, or not-for-profit sectors.

\section{Conflict of interests}

Principal author completed the uniform disclosure form, and all other authors will complete as well upon submission.

\section{Ethical Approval}

This study was approved by the institute's IRB.

\section{REFERENCES}

1. Rochwerg B, Brochard L, Elliott MW, et al. Official ERS/ATS clinical practice guidelines: noninvasive ventilation for acute respiratory failure. Eur Respir J 2017;50(2):1602426. doi: 10.1183/13993003.02426-2016

2. Brochard L, Mancebo J, Wysocki M, et al. Noninvasive ventilation for acute exacerbations of chronic obstructive pulmonary disease. N Engl J Med 1995;333(13):817-22. doi: 10.1056/NEJM1995092833331301

3. Chandra D, Stamm JA, Taylor B, Ramos RM, Satterwhite L. Outcomes of noninvasive ventilation for acute exacerbations of chronic obstructive pulmonary disease in the United States, 1998-2008. 2008;185:152-9. doi: $10.1164 / \mathrm{rccm} .201106-10940 \mathrm{C}$

4. Mosier JM, Sakles JC, Whitmore SP, et al. Failed noninvasive positive-pressure ventilation is associated with an increased risk of intubation-related complications. Ann Intensive Care 2015;5:4. doi: 10.1186/ s13613-015-0044-1

5. Meltem TM, Suna A, Levent K, Remzi A, Figen A, Akkoyunlu ME. Predictors of success and outcome of noninvasive ventilation in acute exacerbations of COPD at A. Chest 2005;128(4):256S. doi: 10.1378/chest.128.4

6. Bhattacharyya CD, Prasad BB, Capt S, Tampi PS, Ramprasad CR. Early predictors of success of non-invasive positive pressure ventilation in hypercapnic respiratory failure. Med J Armed Forces India 2011;67(4):315-19. doi:10.1016/S0377-1237(11)60075-0

7. Lemyze M, Bury Q, Guiot A, et al. Delayed but successful response to noninvasive ventilation in COPD patients with acute hypercapnic respiratory failure. Int J COPD 2017;12:1539-47. doi: 10.2147/COPD. S136241

8. Walkey AJ, Wiener RS. Use of noninvasive ventilation in patients with acute respiratory failure, 2000-2009: a population-based study. Ann Am Thorac Soc 2013;10(1):10-17. doi: 10.1513/AnnalsATS.201206-034OC

9. Corrêa TD, Sanches PR, de Morais LC, Scarin FC, Silva E, Barbas CSV. Performance of noninvasive ventilation in acute respiratory failure in critically ill patients: a prospective, observational, cohort study. BMC Pulm Med 2015;15:144. doi: 10.1186/s12890-015-0139-3

10. Steriade AT, Johari S, Sargarovschi N, et al. Predictors of outcome of noninvasive ventilation in severe COPD exacerbation. BMC Pulm Med 2019;19:131.

11. Duan J, Bai L. Response to "Early prediction of noninvasive ventilation failure in COPD patients: derivation, internal validation, and external 


\section{Ghazala et al.}

validation of a simple risk score." Ann Intensive Care 2019;9(1):140. doi: 10.1186/s13613-019-0614-8

12. Confalonieri M, Garuti G, Cattaruzza MS, et al. A chart of failure risk for noninvasive ventilation in patients with COPD exacerbation. Eur Respir J 2005;25(2):348-55. doi: 10.1183/09031936.05.00085304

13. Vaudan S, Ratano D, Beuret P, Hauptmann J, Contal O, Garin N. Impact of a dedicated noninvasive ventilation team on intubation and mortality rates in severe COPD exacerbations. Respir Care 2015;60(10):1404-8. doi: $10.4187 /$ respcare. 03844

14. Guo Y, Zhang T, Wang Z, et al. Body mass index and mortality in chronic obstructive pulmonary disease. 2016;95(28):e4225.

15. Lambert AA, Putcha N, Drummond MB, et al. Obesity is associated with increased morbidity in moderate to severe COPD. Chest 2017;151(1):6877. doi:10.1016/j.chest.2016.08.1432
16. Wunsch H. Mechanical ventilation in COVID-19: interpreting the current epidemiology. Am J Respir Crit Care Med 2020;202(1):1-4. doi: 10.1164/rccm.202004-1385ED

17. Khilnani GC, Saikia N, Banga A, Sharma SK. Non-invasive ventilation for acute exacerbation of COPD with very high $\mathrm{PaCO}_{2}$ : a randomized controlled trial. Lung India 2010;27(3):125-30. doi: 10.4103/0970-2113.68308

18. Bafadhel M, McKenna S, Terry S, et al. Acute exacerbations of chronic obstructive pulmonary disease: identification of biologic clusters and their biomarkers. Am J Respir Crit Care Med 2011;184(6):662-71. doi: 10.1164/ rccm.201104-0597OC

19. Lopez-Campos JL, Agustí A. Heterogeneity of chronic obstructive pulmonary disease exacerbations: a two-axes classification proposal. Lancet Respir Med 2015;3(9):729-34. doi: 10.1016/S2213-2600(15)00242-8 